1. Brown SL, Gropler RJ, Harris KM. Distinguishing left ventricular aneurysm from pseudoaneurysm. A review of the literature. Chest 1997; 111: 1403-9.

2. Pollak H, Nobis H, Miczoch J. Frequency of left ventricular free wall rupture complicating acute myocardial infarction since the advent of thrombolysis. Am J Cardiol 1994; 74: 184-6.

3. Bates RJ, Beutler S, Resnekov L, et al. Cardiac rupture-challenge in diagnosis and management. Am J Cardiol 1977; 40: 429-47.

4. Hung MJ, Wang CH, Cherng WJ. Unruptured left ventricular pseudoaneurysm following myocardial infarction. Heart 1998; 80: 94-7.

5. Gatewood RP, Nanda NC. Differentiation of left ventricular pseudoaneurysm from true aneurysm with two-dimensional echocardiography. Am J Cardiology 1980; 46: 869-78.

6. Spindola-Franco H, Kronacher N. Pseudoaneurysm of the left ventricule: radiographic and angiocardiographic diagnosis. Radiology 1978; 127: 29-34.

7. Chakraborty RN, Nicholson AA, Alamgir MF. Magnetic Resonance Images of left ventricular pseudoaneurysm. Heart 1998; 80: 101-3.

8. Kumbasar B, Wu KC, Kamel IR, Lima JAC, Bluemke DA. Left ventricular true aneurysm: diagnosis of myocardial viability shown on MR imaging. AJR 2002; 179: 472-4.

\section{Disnea aguda y TAC helicoidal torácico}

\section{Sr. Director:}

El diagnóstico de tromboembolismo pulmonar (TEP) sigue siendo complicado. Supone unos 600.000 nuevos pacientes/año en EE.UU. La angiografía pulmonar (técnica patrón) es invasiva aunque con escasas complicaciones. La gammagrafía pulmonar (GP) de alta probabilidad indica habitualmente su presencia pero pocos pacientes la presentan. Su valor predictivo positivo es del $85-87 \%$. Se altera por la presencia de EPOC o TEP previo. A continuación exponemos la utilidad de la tomografía axial computarizada helicoidal (TACH) en el diagnóstico de TEP.

Mujer de 72 años con neoplasia mamaria izquierda intervenida y tratada con quimioterapia y radioterapia 4 años antes. Dos meses antes sufrió paraparesia secundaria a compresión medular metastásica en L2. Acude por disnea brusca y sudoración. Exp. Física: TA 150/90 mm Hg, taquipnea con $34 \mathrm{rpm}$, taquicardia a $113 \mathrm{lpm}$ y roncus dispersos pulmonares. Hemograma: Hb 11,9 g/L. Bioquímica sérica normal. TP $78 \%$ con TTPa normal. Dímero D positivo [2326 ng/ml (normal 0-500)]. Gasometría arterial: $\mathrm{pO}_{2} 53 \mathrm{~mm} \mathrm{Hg}$ y $\mathrm{pCO}_{2} 27 \mathrm{mmHg}$. EKG: taquicardia sinusal con $\mathrm{S} 1$. Rx. Tórax normal. Se realizó TACH torácica que mostró la presencia de una gran trombo en la bifurcación de la arteria pulmonar extendiéndose por en nacimiento de ambas arterias pulmonares principales (Fig. 1). La GP mostró amplios defectos bilaterales de perfusión con ventilación normal. Se inició anticoagulación con lenta pero buena evolución clínica.

El diagnóstico de TEP sigue siendo difícil. La TACH lo ha revolucionado al permitir realizarlo salvo en los casos de TEP subsegmentarios $(1,2)$. Se requiere la opacificación intensa de las arterias pulmonares. El diagnóstico en las arterias centrales tiene una sensibilidad del $90 \%$ y especificidad del 96\%. Cuando se incluyen vasos subsegmentarios la sensiblidad baja al $67 \%$ y la especificidad al 89\%. La tasa de TEP sugsegmentario aislado es sólo del 6\% (3) y su importancia clínica aún no ha sido claramente definida. Parece existir un riesgo aumentado de recurrencia en los pacientes que lo padecen (4). La TACH es útil para evaluar pacientes con enfermedad cardiopulmonar grave asociada pero la seguridad de retirar anticoagulación si es negativa para TEP aún no está demostrada (5). Con la TACH de múltiples hélices para eliminar artefactos, las arterias pulmonares se pueden estudiar hasta llegar al $5^{\circ}$ orden. Esto implica una mejor probabilidad de

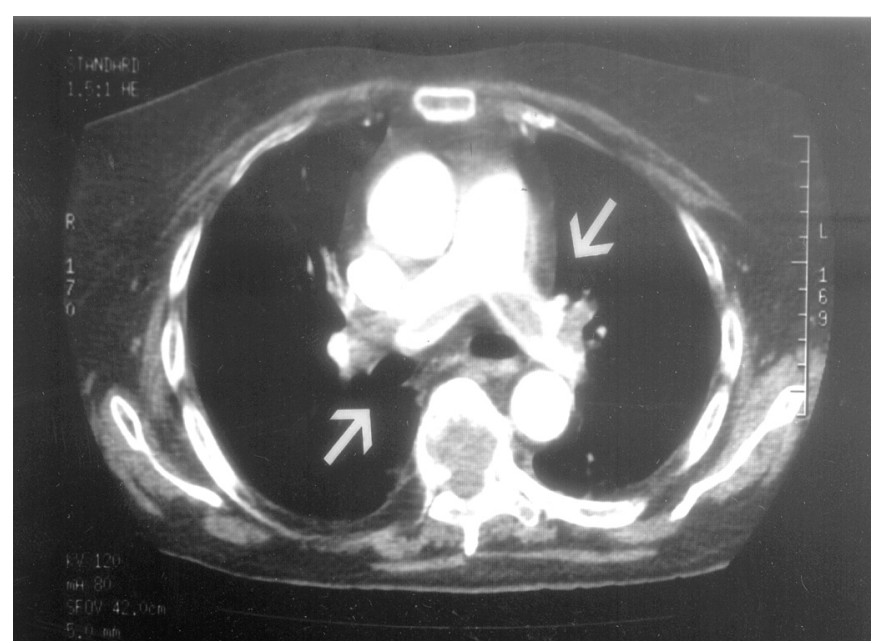

Fig. 1. TAC helicoidal torácico: presencia de un trombo en la bifurcación de arteria pulmonar.

detección de émbolos subsegmentarias (6). Así, la TACH (en especial con fase venosa) puede llegar a ser de primera línea en la evaluación del TEP (7). Una TACH negativa más una ecografía de troncos venosos también negativa incrementa sensibilidad y especificidad para TEP con respecto a la TACH sola. La GP parece más útil en pacientes previamente sanos con radiografía de tórax normal mientras que en pacientes que no cumplen esto sería más útil la TACH (8). En un estudio español se evidenció concordancia entre la GP y la TACH en el 70,3\% de los pacientes (diagnóstico de TEP 13 casos y ausencia 25). En los estudios discordantes se evidenció TEP con GP en 2 pacientes con TAC normal (9). La TACH detecta todos los TEP relevantes y ayuda al diagnóstico diferencial si la GP no es diagnóstica o de baja-intermedia probabilidad. Por otro lado, una determinación rápida de dímero D por ELISA puede reducir la necesidad de realizar una TACH en un 25\%-35\% de casos (10). La TACH es más barata, segura, rápida y fácil de hacer que la arteriografía pulmonar y además permite una mayor accesibilidad diagnóstica para el paciente urgente y en hospitales que no disponen de la posibilidad de realizar GP.

\section{J. A. Díaz Peromingo, R. Puerta Louro, E. Padín Paz, H. Periscal Martínez ${ }^{1}$}

Servicios de Medicina Interna $y^{\prime}$ Urgencias. Hospital Da Barbanza. Riveria. A Coruña

1. Gulsun M, Goodman L, Washington L. Venous thoromboembolic disease: where does multidetector computed tomography fit? Cardiol Clin 2003; 21: 631-638.

2. Weg J. Current diagnoss techniques for pulmonary embolism. Semin Vasc Surg 2000; 13: 182-188.

3. The PIOPED investigators. Value of ventilation/perfusion scan in the acute pulmonary embolism: results of the prospective investigation pulmonary embolism diagnosis (PIOPED). JAMA 1990; 263: 2753-2759.

4. Kearon C. Diagnosis of pulmonary embolism. CMAJ 2003; 168: 183194.

5. Elliott C. Pulmonary embolism diagnosis in hospitalized and intensive care unit patients. Semin Vasc Med 2001; 1: 205-221.

6. Pattynama P, Kuiper J. Second-generation, subsecond multislice computed-tomography: advancing the role of helical CT pulmonary angiography in suspected pulmonary embolism. Semin Vasc Med 2001; 1: 195-204.

7. Srivastava S, Eagleton M, Greenfield L. Diagnosis of pulmonary ambolism with various imaging modalities. Semin Vasc Surg 2004; 17 (2): 173-180. 
8. Novelline R, Rhea J, Rao P, Stuk J. Helical CT in Emergency Radiology. Radiology 1999; 213: 321-339.

9. Mitjavila M, Balsa M, García-Cañamaque L, Gómez-Santos D, Penín F, Torres V, Gambí N y Pey C. TAC helicoidal y gammagrafía de perfusión pulmonar: diagnóstico de tromboembolismo pulmonar en la práctica clínica. Rev Esp Med Nucl 2004; 23: 71-77.

10. Michiels J, Schroyens W, De Backer W, van der Planken M, Hoogsteden H, Pattynama P. Non-invasive exclusion and diagnosis of pulmonary embolism by sequential use of the rapid ELISA D-dimer assay, clinical score and spiral CT. Int Angiol 2003; 22: 1-14.

\section{Onda J de Osborn en el paciente crítico secundaria hipotermia grave accidental}

\section{Sr. Director:}

En 1953 el Dr. John Osborn describe la existencia de ondas J como resultado de una fibrilación ventricular durante una hipotermia experimental (1). La hipotermia en general queda definida cuando la temperatura corporal desciende por debajo de $35^{\circ} \mathrm{C}(2)$. No se conoce exactamente su mecanismo de producción pero su amplitud se relaciona de manera inversa con la temperatura del cuerpo $(1,3)$. Describimos a continuación nuestra experiencia en este ámbito, infrecuente en las áreas urbanas.

Paciente de 68 años de edad con antecedentes médicos de demencia incipiente que requiere ingreso en UCI debido a hipotermia prolongada en su domicilio. A su llegada a nuestra unidad su temperatura es de $26^{\circ} \mathrm{C}$, presenta un bajo nivel de conciencia con GCS de 10 (M6, O3, V1). El análisis inicial realizado revela graves alteraciones en el equilibrio ácido-base con marcada acidosis metabólica en la gasometría venosa $\left(\mathrm{pH} 7,1 ; \mathrm{pO}_{2} 93 \mathrm{~mm}\right.$ $\mathrm{Hg}, \mathrm{pCO}_{2} 47 \mathrm{~mm} \mathrm{Hg}$, exceso base $-14 \mathrm{mmol} / \mathrm{l}, \mathrm{SaO}_{2} 93,8 \%$ ) así como otras alteraciones, como incremento de urea y creatinina elevados (170 y 3,3 mg/dl respectivamente), elevación de las enzimas musculares (LDH 1330 UI/L, CPK 8792 UI/L, MB 180, mioglobina $>40.000$ y troponina I $0,02 \mathrm{ng} / \mathrm{ml}$ ); el resto de la analítica fue normal, sin existencia de otras alteraciones hematológicas ni metabólicas. La radiografía de tórax fue compatible con la normalidad y el TAC cerebral practicado de manera urgente únicamente mostraba signos de atrofia cerebral.

En el trazado ECG inicial aparece la existencia de ondas $\mathrm{J}$ de Osborn de amplitud similar a las ondas $\mathrm{R}$ en las derivaciones de V3 a V6, así como otros hallazgos como bradicardia sinusal, aumento del intervalo QRS y del segmento QTc (Fig. 1). Se presentaron otras arritmias concurrentes a lo largo de la evolución del paciente, como fue un episodio de fibrilación auricular. Durante su recalentamiento progresivo desaparecieron las mencionadas alteraciones logrando la completa normalización del trazado ECG con la recuperación de la temperatura corporal por encima de $36^{\circ} \mathrm{C}$.

Aproximadamente en el $80 \%$ de los pacientes que presentan hipotermia grave cuentan con grandes alteraciones electrocardiográficas, de ellas la más significativa aunque no la más frecuente es la onda J de Osborn, que viene representada en el ECG por una deflexión que se inscribe entre el comienzo del complejo QRS y el inicio del segmento ST $(4,5)$. No se conoce exactamente su mecanismo de producción (aunque se piensa que es debido a un aumento del potasio en el epicardio durante la repolarización ventricular) (10) pero su amplitud se relaciona de manera inversa con la temperatura del cuerpo. A pesar de la espectacularidad de los hallazgos descritos (6-9) se debe tener en cuenta que para lograr la reversión de estas alteraciones, lo más importante es el adecuado y precoz tratamiento de la hipotermia (10), al igual de lo que sucedió en nuestra breve experiencia.

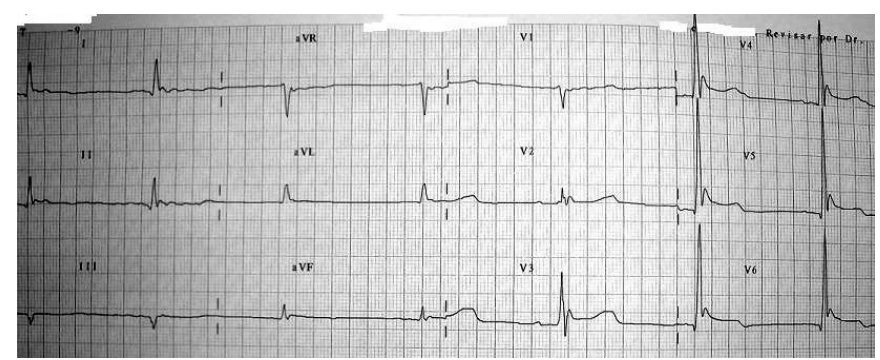

Fig. 1.

\section{B. Obón Azuara, I. Gutiérrez Cía, C. Sánchez Polo', L. Moun- roval $^{2}$}

Servicio de Medicina Intensiva. Hospital Clínico Universitario. Zaragoza. ${ }^{1}$ Servicios de Medicina Intensiva $y{ }^{2}$ Medicina Interna. Hospital Obispo Polanco. Teruel

1. Osborn JJ. Experimental hypotermia respiratory and blood changes in relation to cardiac function. Am J Physiol 1953; 175: 389.

2. Mattu A, Brady WJ, Perron AD. Electrocardiographic manifestations of hypothermia. Am J Emerg Med 2002; 20: 314-26.

3. Okada M, Nishimura F, Yoshino H, Kimura M, Ogino T.The J wave in accidental hypothermia. J Electrocardiol 1983; 16: 23-8.

4. Janssens U, Scheneider B, Hanrath P. Electrocardiographic changes in unintentional hypotermia. Intensive Care Med 1998; 24: 1118-9.

5. Imad A, Alhaddad IA, Khalil M, Brown EJ. Osborn waves of hypotermia. Circulation 2000; 101: E233-44.

6. Nolan J, Soar J. The ECG in Hypotermia. Resucitation 2005; 64: 133-4.

7. Graham CA, McNaughton GW, Wyatt JP. The electrocardiogram in hypothermia. Wilderness Environ Med 2001; 12: 232-5.

8. Spodick DH. Osborne $(\mathrm{J})$ waves in hypothermia. Am J Geriatr Cardiol 2002; 11: 412

9. Ferh T, Straumann EH, Bertel O. Osborn waves of severe hypotermia. Clin Cardiol 2002; 25: 241.

10. Mori J, Krantz MD, Christopher M, Lowery MD. Giant Osborn Waves in hypotermia. NEJM 2005; 352: 184.

\section{Disfunción diastólica con fracción de eyección conservada en una paciente con enfermedad de Paget poliostótica}

\section{Sr. Director:}

En la enfermedad de Paget del hueso se han descrito diversas alteraciones cardíacas (1). La cardiomegalia se asoció hace años a la extensión de la enfermedad ósea (2). También se han descrito hipertrofia ventricular y alteraciones en el electrocardiograma (EKG) asociadas a alteraciones en la conducción (3). La hipertensión arterial se ha diagnosticado en el $25 \%$ de estos pacientes. Durante muchos años la alteración cardiológica típica de la enfermedad de Paget del hueso fue la insuficiencia cardíaca de alto gasto (1-3). Presentamos a continuación un caso de disfunción diastólica con fracción de eyección conservada que respondió muy bien desde el punto de vista sintomático a dosis bajas de un inhibidor de la enzima convertidora de angiotensina.

Mujer que en el año 1992 había sido diagnosticada de una enfermedad de Paget del hueso con afectación de la hemipelvis izquierda. Durante la evolución de su enfermedad la fosfatasa alcalina plasmática ha alcanzado valores entre 450 y 678 UI. En el año 1999 le fue diagnosticado otro foco de actividad pagética en el cuerpo de la tercera vértebra lumbar. Ha sido tratada con calcitonina intramus- 